\title{
A reasoning system for image analysis
}

\author{
Jin Sheng GAO ${ }^{1, a}$, Chang Le ZHOU ${ }^{2,3}$ \\ ${ }^{1}$ Department of philosophy, Xiamen University, Xiamen 361005, China \\ ${ }^{2}$ Department of Cognitive Science and Technology, Xiamen University, Xiamen 361005, China \\ ${ }^{3}$ Fujian Key Lab of the Brain-like Intelligent Systems, Xiamen 361005, China
}

\begin{abstract}
For image analysis in computer, the traditional approach is extracting and transcoding features after image segmentation. However, in this paper, we present a different way to analyze image. We adopt spatial logic technology to establish a reasoning system with corresponding semantic model, and prove its soundness and completeness, and then realize the image analysis in formal way. And it can be applied in artificial intelligence. This is a new attempt and also a challenging approach.
\end{abstract}

\section{Introduction}

The emergence of the digital image analysis technology and its development is only a few decades. But it has deep into our life, such as, artificial intelligence [1], military [2], medical science [3] and other fields [4], and become our main production tools and modern life tools $[5,6]$. By the current conventional methods, the image analysis based on computer is usually done with the following procedures: input high quality image, image segmentation, feature extraction, converting features into quantitative expression, image description, image understanding, and so on $[7,8]$. However, in this paper, we don't take a usual measure to analyse image but spatial logic that is a special technique in topology and modal logic $[9,10]$. We establish a corresponding semantic model and axiomatic system (notation: $S_{I A}$ ), and then realize the image analysis in formal reasoning method. It is not only a new attempt but also a challenging approach.

In order to express our new method systematically, the framework of this paper is roughly as follows: In section 2, we introduce a formal language to express some information which we need in the following system and computer can recognize. And in section 3, we present a semantic model to represent several key operators in following system. After the previous preparation, we establish a corresponding axiom system in section 4 . In the end, we have some discussion in section 5.

\section{Syntax}

Firstly, we present the syntax of $S_{I A}$. By the syntax of $S_{I A}$, we can recognize which formula is the expression of $S_{I A}$. And our reasoning system just to describe what it can express.
Definition 1 (Language $L_{I A}$ ): Given a set of atomic proposition Atom ( $p \in$ Atom), Boolean operators $\{\neg, \wedge$, $\mathrm{V}, \rightarrow, \leftrightarrow\}$, spatial operators $\left\{\oplus_{1}, \oplus_{2}, \oplus_{3}, \oplus_{4}, \oplus_{5}, \oplus_{6}\right.$, $\left.\oplus_{7}, \oplus_{8}, \oplus_{9}\right\} \quad, \quad$ arbitrary propositions $\{\varphi, \psi\}$ and punctuations $\{$ ), , ( $\}$, then the language of $S_{I A}$ (notation: $\left.L_{I A}\right)$ is defined by BNF as follows:

$\varphi$ ::

$=p|\neg \varphi| \varphi$

$\wedge \psi\left|\oplus_{1} \varphi\right| \oplus_{2} \varphi\left|\oplus_{3} \varphi\right| \oplus_{4} \varphi\left|\oplus_{5} \varphi\right| \oplus_{6} \varphi\left|\oplus_{7} \varphi\right| \oplus_{8} \varphi \mid \oplus_{9} \varphi$

Where, the formula $\oplus_{1} \varphi$ means " $\varphi$ is valid in the horizontal rightward", the formula $\oplus_{2} \varphi$ stands for " $\varphi$ is valid in the upper right", the formula $\oplus_{3} \varphi$ stands for " $\varphi$ is valid in the lower right", the formula $\oplus_{4} \varphi$ means " $\varphi$ is valid in the overlapping point", the formula $\oplus_{5} \varphi$ stands for " $\varphi$ is valid in the vertical upward", the formula $\oplus_{6} \varphi$ means " $\varphi$ is valid vertical downward", $\oplus_{7} \varphi$ means " $\varphi$ is valid in the horizontal leftward", the formulas $\oplus_{8} \varphi$ and $\oplus_{9} \varphi$ stand for " $\varphi$ is valid in the upper left" and " $\varphi$ is valid in the lower left" respectively.

In $S_{I A}$, we take operators of negation $(\neg)$ and conjunction $(\Lambda)$ as basic Boolean connectives. And the other Boolean connectives can be abbreviated as usual, such as $\varphi \vee \psi:=\neg(\varphi \wedge \psi), \varphi \rightarrow \psi:=\neg(\varphi \wedge \neg \psi)$ and $\varphi \leftrightarrow \psi:=\neg(\varphi \wedge \neg \psi) \wedge \neg(\neg \varphi \wedge \psi)$. The binding force of Boolean connectives also as usual, i.e., negation $(\neg)$ has a stronger binding force than conjunction $(\wedge)$ or disjunction $(\vee)$ or implication $(\rightarrow)$ or bi-implication $(\leftrightarrow)$. And spatial operators $\oplus_{i}(i \in[1,9])$ has the same binding force as negation $(\neg)$. Therefore, if $L_{I A}$ does not involve other symbols like $\oplus_{i}(i \in[1,9])$, then $S_{I A}$ has nothing to do only to describe general proposition independent space location. The $L_{I A}$ not only describes the relationship between image points, but also can express the proposition of relationship between any two points in the image.

\footnotetext{
${ }^{\mathrm{a} C}$ Corresponding author: Hi.Jackson@163.com
} 


\section{Semantic}

Definition 2 (The Frame $\mathcal{F}_{I A}$ ): Given an ordered pair $<X, Y, \prec_{x}, \prec_{y}>$, then $\mathcal{F}_{I A}=<X, Y, \prec_{x}, \prec_{y}>$ is a frame of $S_{I A}$, if and only if, $X$ is a nonempty set of horizontal points, $Y$ is a nonempty set of vertical points, and $\prec_{x}, \prec_{y}$ are binary order relation on $X$ and $Y$.

In theory, image domain can be infinite, but in reality, agent visual is blame, therefore the $x$ axis and $y$ axis value is bounded. For convenience, we take $H_{x}$ and $V_{y}$ to represent horizontal and vertical orientation respectively. Then, we have $X=\left[-H_{x},+H_{x}\right]$ and $Y=\left[-V_{y},+V_{y}\right]$. More specifically, for any $x_{1}, x_{2} \in X, x_{1} \prec_{x} x_{2}$ represents " $x_{1}$ is in the left of $x_{2}$ ". Similarly, $y_{1}, y_{2} \in Y$, if $y_{1} \prec_{y} y_{2}$ represents " $y_{1}$ is in the down of $y_{2}$ ".

Definition 3 (The Model $\boldsymbol{M}_{\boldsymbol{I A}}$ ): Given a four tuples $\left\langle X, Y, \prec_{x}, \prec_{y}, V\right\rangle$, then $\left\langle X, Y, \prec_{x}, \prec_{y}, V\right\rangle$ is the model of $S_{I A}$ (notation: $\mathcal{M}_{I A}$ ), if and only if, < $X, Y, \prec_{x}, \prec_{y}>$ is a frame of $S_{I A}, V$ is an assignment function on $\mathcal{F}_{I A}$. And $\left(\mathcal{M}_{I A},(x, y)\right)$ is a notation of point model which means just to consider a point $(x, y)(x \in$ $X, y \in Y)$ that belongs to model $\mathcal{M}_{I A}$.

Specifically, the point model is a major tool for measuring whether a formula $\varphi \in L_{I A}$ is satisfiable on point $s$ or not. Sometimes in order to convenient, the parentheses of point model can be omitted in the case of no confusion.

Definition 4 (Truth): Let $\varphi, \psi \in L_{I A}$ be arbitrary formulas, $\left(\mathcal{M}_{I A},(x, y)\right)$ be a point model of $S_{I A}$, then $\varphi$ is true on $(x, y)$ in $\mathcal{M}_{I A}$ (let $\left(\mathcal{M}_{I A},(x, y)\right) \vDash \varphi$ be a notation for it) can be recursively defined as follows:

(1) $\left(\mathcal{M}_{I A},(x, y)\right) \vDash p$ iff $p \in V((x, y))$;

(2) $\left(\mathcal{M}_{I A},(x, y)\right) \vDash \neg \varphi$ iff $\left(\mathcal{M}_{I A},(x, y)\right) \not \models \varphi$;

(3) $\left(\mathcal{M}_{I A},(x, y)\right) \vDash \varphi \wedge \psi$ iff $\left(\mathcal{M}_{I A},(x, y)\right) \vDash \varphi$ and $\left(\mathcal{M}_{I A},(x, y)\right) \vDash \psi$;

(4) $\left(\mathcal{M}_{I A},(x, y)\right) \vDash \oplus_{1} \varphi$ iff for all $x_{1} \in X$, if $x \prec_{x} x_{1}$, then $\left(\mathcal{M}_{I A},\left(x_{1}, y\right)\right) \vDash \varphi$;

(5) $\left(\mathcal{M}_{I A},(x, y)\right) \vDash \oplus_{2} \varphi$ iff for all $x_{1} \in X, y_{1} \in Y$, if $x \prec_{x} x_{1}$ and $y \prec_{y} y_{1}$, then $\left(\mathcal{M}_{I A},\left(x_{1}, y_{1}\right)\right) \vDash \varphi$;

(6) $\left(\mathcal{M}_{I A},(x, y)\right) \vDash \oplus_{3} \varphi$ iff for all $\mathrm{x}_{1} \in \mathrm{X}, \mathrm{y}_{1} \in \mathrm{Y}$, if $x \prec_{x} x_{1}$ and $y_{1} \prec_{y} y$, then $\left(\mathcal{M}_{I A},\left(x_{1}, y_{1}\right)\right) \vDash \varphi$;

(7) $\left(\mathcal{M}_{I A},(x, y)\right) \vDash \oplus_{4} \varphi$ iff $\left(\mathcal{M}_{I A},(x, y)\right) \vDash \varphi$;

(8) $\left(\mathcal{M}_{I A},(x, y)\right) \vDash \oplus_{5} \varphi$ iff for all $y_{1} \in Y$, if $y \prec_{y} y_{1}$, then $\left(\mathcal{M}_{I A},\left(x, y_{1}\right)\right) \vDash \varphi$;

(9) $\left(\mathcal{M}_{I A},(x, y)\right) \vDash \oplus_{6} \varphi$ iff for all $y_{1} \in Y$, if $y_{1} \prec_{y} y$, then $\left(\mathcal{M}_{I A},\left(x, y_{1}\right)\right) \vDash \varphi$;

(10) $\left(\mathcal{M}_{I A},(x, y)\right) \vDash \oplus_{7} \varphi$ iff for all $x_{1} \in X$, if $x_{1} \prec_{x} x$, then $\left(\mathcal{M}_{I A},\left(x_{1}, y\right)\right) \vDash \varphi$;

(11) $\left(\mathcal{M}_{I A},(x, y)\right) \vDash \oplus_{8} \varphi$ iff for all $x_{1} \in X, y_{1} \in Y$, if $x_{1} \prec_{x} x$ and $y \prec_{y} y_{1}$, then $\left(\mathcal{M}_{I A},\left(x_{1}, y_{1}\right)\right) \vDash \varphi$;

(12) $\left(\mathcal{M}_{I A},(x, y)\right) \vDash \oplus_{9} \varphi$ iff for all $x_{1} \in X, y_{1} \in Y$, if $x_{1} \prec_{x} x$ and $y_{1} \prec_{y} y$, then $\left(\mathcal{M}_{I A},\left(x_{1}, y_{1}\right)\right) \vDash \varphi$.

For the definition 4(1), (2) and (3), their semantic definitions are the same as usual definitions in other formal systems. And for the others, in order to make more detail and visual, we will explain the semantic definitions of above with figures one by one. At the same time, in this process, it shows $S_{I A}$ is how to characterize the image analysis by computer.
Firstly, we purposively extract some points in image (for instance, A, B, C, D, E, F, and G), such as figure 1 . Secondly, given arbitrary real number $i \in\left[-H_{x},+H_{x}\right]$ or $\left[-V_{y},+V_{y}\right]$ (for example, $-e,-d,-c,-b,-a, 0, a, b, c, d, e$ and $-e<-d<-c<$ $\left.-b<-a<0<a<b<c<d \prec e\left(<\in\left\{\prec_{x}, \prec_{y}\right\}\right) \quad\right)$, then we can draw the coordinates with real numbers on $X$ axis and $Y$ axis. Thirdly, the coordinates is placed on the image in the middle, and then it becomes the figure that $\mathrm{A}=(a, d), \mathrm{B}=(e, d), \mathrm{C}=(-b, b), \mathrm{D}=(c, b), \mathrm{E}=(-d,-b)$ and $\mathrm{F}=\mathrm{G}=(c,-b)$. Next, we analyze every semantic definition above in the following figures.

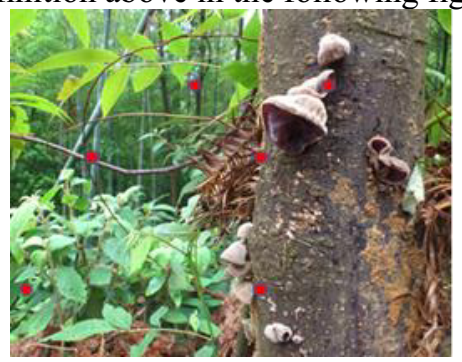

Figure 1. Extract the feature points

In order to highlight the relationship between point and point, the following coordinates omit the background image. For the definition 4(4), it means two points that are $(x, y)$ and $\left(x_{1}, y_{1}\right)$ in image have the relationship of horizontal rightward. And one other thing to note is the $y$ in $(x, y)$ is equal to $y_{1}$ in $\left(x_{1}, y_{1}\right)$. They can be depicted in the following figure 2 . As we can see, $a \prec_{x} e$, $-b \prec_{x} c,-d \prec_{x} c$ and $y=y_{1}=d, y=y_{1}=b$, $y=y_{1}=-b$, therefore $\mathrm{B}, \mathrm{D}, \mathrm{F}$ and $\mathrm{G}$ is respectively in the horizontal rightward of $\mathrm{A}, \mathrm{C}$ and $\mathrm{E}$. In other words, $\oplus_{1} \varphi$ is valid in point $\mathrm{A}=(a, d)$ if $\varphi$ exist in point $\mathrm{B}=(e, d), \oplus_{1} \varphi$ is valid in point $\mathrm{C}=(-b, b)$ if $\varphi$ exist in point $\mathrm{D}=(c, b)$, and $\oplus_{1} \varphi$ is valid in point $\mathrm{E}=(-d,-b)$ if $\varphi$ exist in points $\mathrm{F}$ and $\mathrm{G}=(c,-b)$.

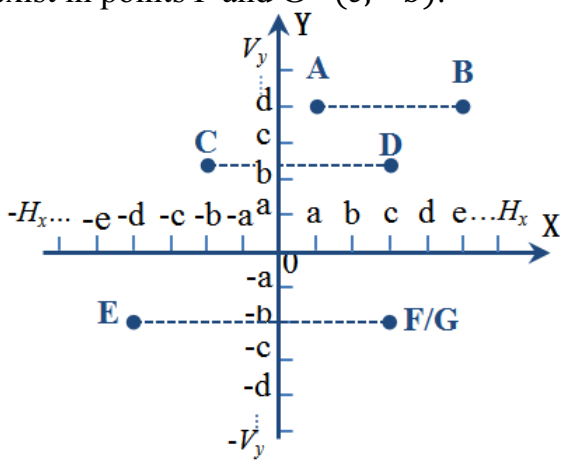

Figure 2.The relationships of horizontal rightward and horizontal leftward

For the definition 4(5), it means " $\varphi$ is valid in the upper right of $(x, y)$ ". As we can see in figure 3, $\mathrm{A}=(a, d)$ is in the upper right of $\mathrm{C}=(-b, b)$ since $b \prec_{y} d$ and $-b \prec_{x} a$. Similarly, $\mathrm{A}=(a, d)$, $\mathrm{B}=(e, d), \mathrm{C}=(-b, b)$ and $\mathrm{D}=(c, b)$ is in the upper right of $\mathrm{E}=(-d,-b)$, and $\mathrm{B}=(e, d)$ is in the upper right of $\mathrm{D}=(c, b)$ and $\mathrm{F} / \mathrm{G}=(c,-b)$. Hence, $\oplus_{2} \varphi$ is valid in points $\mathrm{C}=(-b, b), \mathrm{E}=(-d,-b), \mathrm{D}=(c, b)$ and $\mathrm{F} / \mathrm{G}=(c,-b)$ if $\varphi$ is existing in points $\mathrm{A}=(a, d)$, $\mathrm{B}=(e, d), \mathrm{C}=(-b, b), \mathrm{D}=(c, b)$ and $\mathrm{B}=(e, d)$ respectively. 


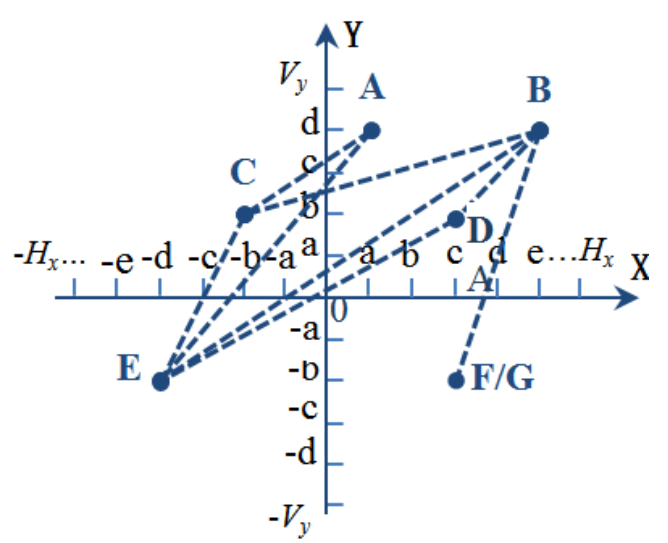

Figure 3. The relationships of upper right and lower left

For the definition $4(6)$, it means " $\varphi$ is valid in the lower right of $(x, y)$ ". We show it in figure $4, \mathrm{D}=(c, b)$ and $\mathrm{F} / \mathrm{G}=(c,-b)$ is in the lower right of $\mathrm{A}=(a, d)$ and $\mathrm{C}=(-b, b)$ respectively for $\left(a \prec_{x} c, b \prec_{y} d\right)$ and $\left(-b \prec_{x} c,-b \prec_{y} b\right)$. Similarly, Hence, $\oplus_{3} \varphi$ is valid in points $\mathrm{A}=(a, d)$ and $\mathrm{C}=(-b, b)$ if $\varphi$ is existing in points $\mathrm{D}=(c, b)$ and $\mathrm{F} / \mathrm{G}=(c,-b)$ respectively.

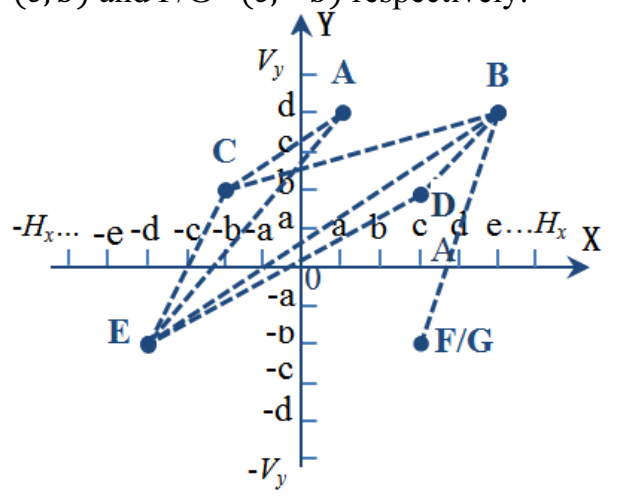

Figure 4. The relationships of lower right and upper left

For the definition $4(7)$, it means " $\varphi$ is valid in $\left(x_{1}, y_{1}\right)$ that is an overlapping point of $(x, y)$ ". It can be represented in figure 5 , since $\mathrm{F}=\mathrm{G}=(c,-b), \oplus_{4} \varphi$ and $\varphi$ are valid in points $\mathrm{F}$ and $\mathrm{G}=(c,-b)$.

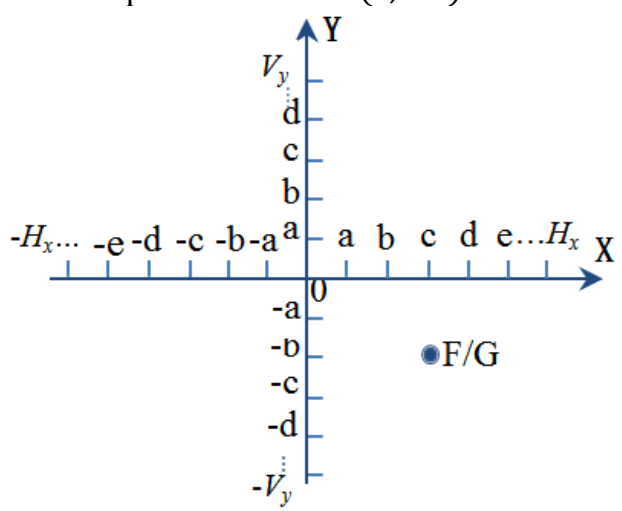

Figure 5.The relationship of overlapping points

For the definition 4(8), it means " $\varphi$ is valid in the vertical upward of $(x, y)$ ". And as shown in figure 6 , they are mirror image relationship for the definitions $4(8)$ and (9). It is easy to find $\oplus_{5} \varphi$ is valid in $\mathrm{D}=(c, b)$ if $\varphi$ is existing in $\mathrm{F} / \mathrm{G}=(c,-b)$, and similarly $\oplus_{6} \varphi$ is valid in $\mathrm{F} / \mathrm{G}=(c,-b)$ if $\varphi$ is existing in $\mathrm{D}=(c, b)$.
For the definition 4(10), as shown in figure $2, \oplus_{7} \varphi$ is a mirror image of $\oplus_{1} \varphi$, and it means " $\oplus_{7} \varphi$ is valid in $(x, y)$ if $\varphi$ is valid in the horizontal leftward of $(x, y)$ ". Similarly, $\oplus_{8} \varphi$ and $\oplus_{9} \varphi$ are mirrored to $\oplus_{3} \varphi$ and $\oplus_{2} \varphi$.

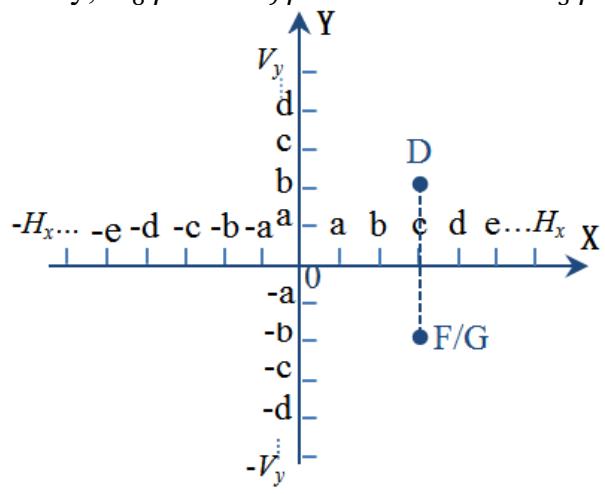

Figure 6. The relationships of vertical upward and vertical downward

\section{The axiomatization of $S_{I A}$ and its theorems}

\subsection{Axiomatization}

Definition 5 (Axiomatization): Let $p \in$ Atom be an atomic proposition, $\varphi, \psi \in L_{I A}$ be arbitrary formulas, and $i \in\{1,2,3,4,5,6,7,8,9\}$, then the axiomatization of $S_{I A}$ consists of the following axiom models and inference rules:
$(\mathrm{AM} 1) \oplus_{\mathrm{i}}(\varphi \rightarrow \psi) \rightarrow\left(\oplus_{\mathrm{i}} \varphi \rightarrow \oplus_{i} \psi\right)$
$(\mathrm{AM} 2) \oplus_{i} \varphi \wedge \oplus_{i} \psi \rightarrow \oplus_{i}(\varphi \wedge \psi)$
$(\mathrm{AM} 3) \oplus_{1} \oplus_{7} \varphi \rightarrow \oplus_{7} \varphi$
$(\mathrm{AM} 4) \oplus_{7} \oplus_{1} \varphi \rightarrow \oplus_{1} \varphi$
(AM5) $\oplus_{5} \oplus_{6} \varphi \rightarrow \oplus_{6} \varphi$
$(\mathrm{AM} 6) \oplus_{6} \oplus_{5} \varphi \rightarrow \oplus_{5} \varphi$
$(\mathrm{AM} 7) \oplus_{2} \oplus_{9} \varphi \rightarrow \oplus_{9} \varphi$
$(\mathrm{AM} 8) \oplus_{9} \oplus_{2} \varphi \rightarrow \oplus_{2} \varphi$
$(\mathrm{AM} 9) \oplus_{3} \oplus_{8} \varphi \rightarrow \oplus_{8} \varphi$
(AM10) $\oplus_{8} \oplus_{3} \varphi \rightarrow \oplus_{3} \varphi$
$(\mathrm{AM} 11) \oplus_{1} \oplus_{2} \varphi \leftrightarrow \oplus_{2} \oplus_{1} \varphi$
$(\mathrm{AM} 12) \oplus_{1} \oplus_{3} \varphi \leftrightarrow \oplus_{3} \oplus_{1} \varphi$
$(\mathrm{AM} 13) \oplus_{1} \oplus_{4} \varphi \rightarrow \oplus_{1} \varphi$
$(\mathrm{AM} 14) \oplus_{1} \oplus_{5} \varphi \leftrightarrow \oplus_{5} \oplus_{1} \varphi$
$(\mathrm{AM} 15) \oplus_{1} \oplus_{6} \varphi \leftrightarrow \oplus_{6} \oplus_{1} \varphi$
(AM16) $\oplus_{1} \oplus_{8} \varphi \leftrightarrow \oplus_{8} \oplus_{1} \varphi$
$(\mathrm{AM} 17) \oplus_{1} \oplus_{9} \varphi \leftrightarrow \oplus_{9} \oplus_{1} \varphi$
$(\mathrm{AM} 18) \oplus_{2} \oplus_{3} \varphi \leftrightarrow \oplus_{3} \oplus_{2} \varphi$
$(\mathrm{AM} 19) \oplus_{2} \oplus_{4} \varphi \rightarrow \oplus_{2} \varphi$
$(\mathrm{AM} 20) \oplus_{2} \oplus_{5} \varphi \leftrightarrow \oplus_{5} \oplus_{2} \varphi$
$(\mathrm{AM} 21) \oplus_{2} \oplus_{6} \varphi \leftrightarrow \oplus_{6} \oplus_{2} \varphi$
$(\mathrm{AM} 22) \oplus_{2} \oplus_{7} \varphi \leftrightarrow \oplus_{7} \oplus_{2} \varphi$
$(\mathrm{AM} 23) \oplus_{2} \oplus_{8} \varphi \leftrightarrow \oplus_{8} \oplus_{2} \varphi$
$(\mathrm{AM} 24) \oplus_{3} \oplus_{4} \varphi \rightarrow \oplus_{3} \varphi$
$(\mathrm{AM} 25) \oplus_{3} \oplus_{5} \varphi \leftrightarrow \oplus_{5} \oplus_{3} \varphi$
$(\mathrm{AM} 26) \oplus_{3} \oplus_{6} \varphi \leftrightarrow \oplus_{6} \oplus_{3} \varphi$
$(\mathrm{AM} 27) \oplus_{3} \oplus_{7} \varphi \leftrightarrow \oplus_{7} \oplus_{3} \varphi$
$(\mathrm{AM} 28) \oplus_{3} \oplus_{9} \varphi \leftrightarrow \oplus_{9} \oplus_{3} \varphi$
$(\mathrm{AM} 29) \oplus_{4} \oplus_{1} \varphi \rightarrow \oplus_{1} \varphi$
$(\mathrm{AM} 30) \oplus_{4} \oplus_{5} \varphi \rightarrow \oplus_{5} \varphi$
$(\mathrm{AM} 31) \oplus_{4} \oplus_{6} \varphi \rightarrow \oplus_{6} \varphi$ 


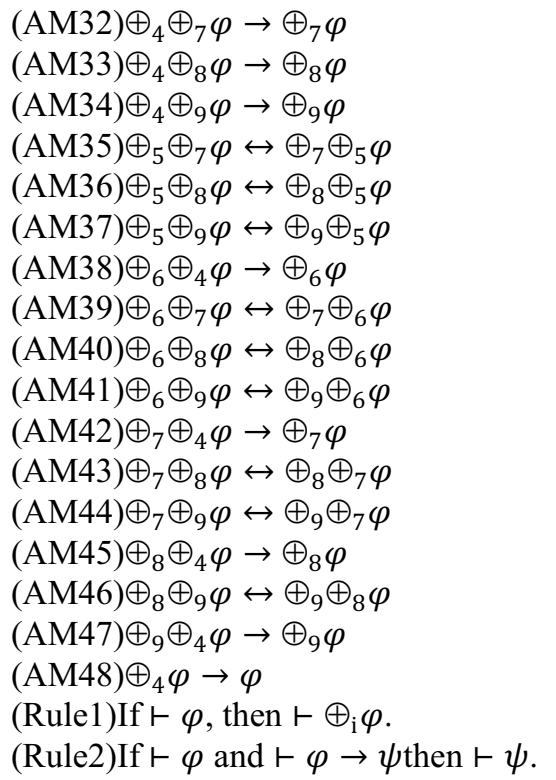

\subsection{Soundness and completeness}

Theorem 1 (Soundness): Let $\varphi \in L_{I A}$ be arbitrary formula, if $\vdash \varphi$ then $\vDash \varphi$.

Proof. It is easy to show all the axioms is valid on the frame, and (Rule1) and (Rule2) are closed under the frame. Thus, $\mathrm{S}_{\mathrm{IA}}$ is soundness.

Definition 6 (Canonical Model): Let < $X^{C}, Y^{C}, \prec_{x}^{C},<_{y}^{C}, V^{C}>$ be the canonical model of $S_{I A}$ (notation: $\mathcal{M}_{I A}^{C}$ ), if and only if, (i) $X^{C}, Y^{C}=$ $\left\{F^{m} \mid F^{m}\right.$ is a maximal consistent formula set of $\left.S_{I A}\right\}$, (ii) $F^{m} \prec_{x}^{C} F^{m^{\prime}}$ iff $\oplus_{i}{ }^{-} F^{m} \subseteq F^{m^{\prime}}, F^{m} \prec_{y}^{C} F^{m^{\prime}}$ iff $\oplus_{i}{ }^{-} F^{m} \subseteq$ $F^{m^{\prime}}$, (iii) $V^{C}(p)=\left\{F^{m} \in X^{C}\right.$ and $\left.Y^{C} \mid p \in F^{m}\right\}$.

Theorem 2: Let Form $\left(L_{I A}\right)$ be the formula set of $S_{I A}$, $F \subseteq \operatorname{Form}\left(L_{I A}\right)$ be an arbitrary subset, $\varphi \in \operatorname{Form}\left(L_{I A}\right)$ be an arbitrary formula of $S_{I A}$, if $F$ is consistent and $\neg \oplus_{i} \varphi \in F$, then $\oplus_{i}{ }^{-} F \cup\{\neg \varphi\}$ is consistent.

Proof. By the structured induction on $\varphi \in \operatorname{Form}\left(L_{I A}\right)$, and the axioms and inference rules of $S_{I A}$, it can be proved.

Theorem 3: Let Form $\left(L_{I A}\right)$ be the formula set of $S_{I A}$, $F \subseteq \operatorname{Form}\left(L_{I A}\right)$ be arbitrary subsets, $\varphi \in \operatorname{Form}\left(L_{I A}\right)$ be an arbitrary formula of $S_{I A}$, if $\neg \oplus_{i} \varphi \in F$, then there are $F^{\prime} \subseteq \operatorname{Form}\left(L_{I A}\right)$ that is $F \prec F^{\prime}\left(\prec \in\left\{\prec_{x}, \prec_{y}\right\}\right)$ and $\neg \varphi \in F^{\prime}$.

Proof.Assume $\neg \oplus_{i} \varphi \in F$, then by "theorem 2", we have $\oplus_{i}{ }^{-} F \cup\{\neg \varphi\}$ is consistent. And by the property theorem of consistent set, it can be expanded to a maximal consistent set $F^{\prime}$. Thus $\neg \varphi \in F^{\prime}$.

Theorem 4: Let $\mathcal{M}_{I A}^{C}$ be the canonical model of $S_{I A}$, $\psi \in \operatorname{Form}\left(L_{I A}\right)$ be an arbitrary formula of $S_{I A}$, then existing $\left(\mathcal{M}_{I A}^{C}, F_{x}^{m}, F_{y}^{m}\right) \vDash \psi$ iff $\psi \in\left(F_{x}^{m} \cap F_{y}^{m}\right)$.

Proof.Consider the induction on $\psi$.

(i) Assume $\psi$ is $p \in \operatorname{Form}\left(L_{I A}\right)$. By $\mathcal{M}_{I A}^{C}$, that $F_{x}^{m}, F_{y}^{m} \in$ $V^{C}(p)$ iff $p \in\left(F_{x}^{m} \cap F_{y}^{m}\right)$.

(ii) Assume $\psi$ is $\neg \varphi$. Then $\left(\mathcal{M}_{I A}^{C}, F_{x}^{m}, F_{y}^{m}\right) \vDash \neg \varphi$, by "definition 4", iff $\left(\mathcal{M}_{I A}^{C}, F_{x}^{m}, F_{y}^{m}\right) \not \models \varphi$. By introduction hypothesis, iff $\varphi \notin\left(F_{x}^{m} \cap F_{y}^{m}\right)$. And by property theorem of consistent set, we have $\neg \varphi \in\left(F_{x}^{m} \cap F_{y}^{m}\right)$.

(iii) Assume $\psi$ is $\varphi_{1} \wedge \varphi_{2}$. Then $\left(\mathcal{M}_{I A}^{C}, F_{x}^{m}, F_{y}^{m}\right) \vDash \varphi_{1} \wedge$ $\varphi_{2}$, by "definition 4", iff $\left(\mathcal{M}_{I A}^{C}, F_{x}^{m}, F_{y}^{m}\right) \vDash \varphi_{1}$ and $\left(\mathcal{M}_{I A}^{C}, F_{x}^{m}, F_{y}^{m}\right) \vDash \varphi_{2}$. By introduction hypothesis, iff $\varphi_{1} \in\left(F_{x}^{m} \cap F_{y}^{m}\right)$ and $\varphi_{2} \in\left(F_{x}^{m} \cap F_{y}^{m}\right)$. And by property theorem of consistent set, we have $\varphi_{1} \wedge \varphi_{2} \in\left(F_{x}^{m} \cap F_{y}^{m}\right)$. (iv) Assume $\psi$ is $\oplus_{1} \varphi$. Suppose $\oplus_{1} \varphi \in\left(F_{x}^{m} \cap F_{y}^{m}\right)$. By "definition 6", for $F_{x}^{m^{\prime}} \in X^{C}$ and $F_{y}^{m^{\prime}} \in Y^{C}$, if $F_{x}^{m} \prec_{x}^{C} F_{x}^{m^{\prime}}$ and $F_{y}^{m} \prec_{y}^{C} F_{y}^{m^{\prime}}$, then $\varphi \in\left(F_{x}^{m^{\prime}} \cap F_{y}^{m^{\prime}}\right)$. By induction hypothesis, for any $F_{x}^{m^{\prime}} \in X^{C}$ and $F_{y}^{m^{\prime}} \in Y^{C}$, if $F_{x}^{m} \prec_{x}^{C} F_{x}^{m^{\prime}}$ and $F_{y}^{m} \prec_{y}^{C} F_{y}^{m^{\prime}}$, then $\left(\mathcal{M}_{I A}^{C}, F_{x}^{m^{\prime}}, F_{y}^{m^{\prime}}\right) \vDash \varphi$. And by "definition 4", $\left(\mathcal{M}_{I A}^{C}, F_{x}^{m}, F_{y}^{m}\right) \vDash \oplus_{1} \varphi$. Suppose $\oplus_{1} \varphi \notin\left(F_{x}^{m} \cap F_{y}^{m}\right)$. By property theorem of consistent set, we have $\neg \oplus_{1} \varphi \in\left(F_{x}^{m} \cap F_{y}^{m}\right)$. And by "theorem 3", there are $F_{x}^{m^{\prime}} \in X^{C}$ and $F_{y}^{m^{\prime}} \in Y^{C}$, such that $\neg \varphi \in$ $\left(F_{x}^{m^{\prime}} \cap F_{y}^{m^{\prime}}\right)$. By property theorem of consistent set, $\varphi \notin\left(F_{x}^{m^{\prime}} \cap F_{y}^{m^{\prime}}\right)$. And induction hypothesis, $\left(\mathcal{M}_{I A}^{C}, F_{x}^{m^{\prime}}, F_{y}^{m^{\prime}}\right) \not \varphi \quad$. By “definition 4", $\left(\mathcal{M}_{I A}^{C}, F_{x}^{m}, F_{y}^{m}\right) \not \# \oplus_{1} \varphi$.

(v) Similarly, we can prove the other cases of $\psi$ is $\oplus_{i} \varphi(i \in\{2,3,4,5,6,7,8,9\})$, there is $\left(\mathcal{M}_{I A}^{C}, F_{x}^{m}, F_{y}^{m}\right) \vDash$ $\oplus_{i} \varphi$ iff $\oplus_{i} \varphi \in\left(F_{x}^{m} \cap F_{y}^{m}\right)$.

Theorem 5(Completeness): Let $\varphi \in L_{I A}$ be arbitrary formula, if $\vDash \varphi$ then $\vdash \varphi$.

Proof. Let $\mathcal{M}_{I A}^{C}=<X^{C}, Y^{C}, \prec_{x}^{C}, \prec_{y}^{C}, V^{C}>$ be the canonical model of $S_{I A}, F_{x}^{m} \in X^{C}$ and $F_{y}^{m} \in Y^{C}$ be any maximal consistent sets. Assume $\forall \varphi$. By property theorem of consistent set, we have $\varphi \notin\left(F_{x}^{m} \cap F_{y}^{m}\right)$. And by "theorem 4 ", $\left(\mathcal{M}_{I A}^{C}, F_{x}^{m}, F_{y}^{m}\right) \not \models \varphi$.

\section{Discussion}

We have established a reasoning system $S_{I A}$ that can be used in the computer image analysis. In this system, people just need to put the image under a computer with a camera, or through computer scanning, and then the computer can according to the coordinates of system analyzes the relationship between any pixels or compositions in this image. Moreover, it can be carried out a series of location analysis, so as to achieve the expected effect of image processing in computer. We just need to compile the system in the form of a language as a programming language that can be systematically reasoning. However, we must earnestly point out this system still have some shortcomings, for instance, it fails to directly exhibit what specific area have but through indirect and tedious iteration by different operators. To solve these defects, apparently, is our future work.

\section{Acknowledgements}

This work was supported in part by the National Natural Science Foundation of China under Grant Nos.61273338, and in part by the Major Program of National Social Science Foundation of China under Grant 11\&ZD088. 


\section{References}

1. S. S. Rautaray, A. Agrawal. Vision based hand gesture recognition for human computer interaction: a survey. Artificial Intelligence Review, 43(1), 1-54, (2015)

2. Y. Ahn, S. Lee. Hit Rate Prediction Algorithm for Laser Guided Bombs Using Image Processing. KIISE Transactions on Computing Practices, 21(3), 247-256, (2015)

3. X. Zhang, W. Liu, M. Dundar, S. Badve, S. Zhang. Towards large-scale histopathological image analysis: Hashing-based image retrieval.Medical Imaging, IEEE Transactions on, 34(2), 496-506, (2015)

4. B. Rieger, R. Nieuwenhuizen, S. Stallinga. Image Processing and Analysis for Single-Molecule Localization Microscopy: Computation for nanoscale imaging. Signal Processing Magazine, IEEE, 32(1), 49-57, (2015)

5. E. Boix, A. Ferrer, S. Fernandez, X. Sellart. Vehicle Guiding System Through Image Processing in Crash and Misuse Tests. In Proceedings of SAE-China Congress 2014: Selected Papers (Springer Berlin Heidelberg, 411-424, 2014)

6. E. Tabassi, P. Grother. Fingerprint image quality.In Encyclopedia of Biometrics (Springer US, 635-643, 2015).

7. J. C. Russ. The Image Processing Handbook. (CRC Press, 269-629, 2007)

8. M. Sonka, V. Hlavac, R. Boyle. Image processing, analysis, and machine vision (Cengage Learning,1537, 2015)

9. M. Aiello, I. Pratt-Hartmann, J. van Benthem (Eds.). Handbook of spatial logics (Vol. 4)(Springer, 217-291, 2007)

10. P. Blackburn, M. De Rijke, Y. Venema. Modal Logic (Vol. 53) (Cambridge University Press, 50-426, 2001) 